\title{
Continuous Renal Replacement Therapy Clearance Rate for Salicylate Toxicity in Multi-System Trauma*
}

\author{
Daniel Silverman $^{1}$, L. Keith French ${ }^{2,3}$, Robert G. Hendrickson ${ }^{2,3}$ \\ ${ }^{1}$ School of Medicine, Oregon Health \& Science University, Portland, USA; ${ }^{2}$ Department of Emergency Medicine, Oregon Health \& \\ Science University, Portland, USA; ${ }^{3}$ Oregon Poison Center, Portland, USA. \\ Email: frenchk@ohsu.edu
}

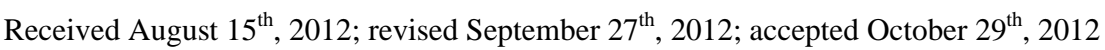

\begin{abstract}
The management of patients with concomitant xenobiotic toxicity and multisystem trauma can be complex. While hemodialysis is generally the modality of choice for extracorporeal elimination of salicylates, the potential for large volume shifts and hypotension may pose a risk in patients with traumatic brain injury. An alternative therapy to hemodialysis is continuous renal replacement therapy, which has slower clearance rates than hemodialysis, but has decreased adverse effects in cases of traumatic brain injury. However, there are few published reports of clearance rates of salicylates using continuous renal replacement therapy. We report a case of multisystem trauma with concomitant intentional salicylate overdose in which continuous renal replacement therapy was employed. The salicylate clearance rate that was obtained in this case was $7.5 \mathrm{~mL} /$ minute using continuous veno-venous hemodiafiltration, a form of continuous renal replacement therapy.
\end{abstract}

Keywords: CRRT; Clearance Rate; Salicylate Toxicity; Multisystem Trauma

\section{Introduction}

Continuous renal replacement therapy (CRRT) was first introduced over 20 years ago as an alternative to hemodialysis (HD) for management of unstable and critically ill patients [1]. Joy et al. have characterized all forms of CRRT in a primer that describes the physics, methodology, and employment [2]. Continuous veno-venous hemodiafiltration (CVVHD, a form of CRRT) has since been described for use in management of toxicity in hemodynamically unstable patients who cannot tolerate dialysis, although this point remains controversial [3,4]. A major advantage of CVVHD is ease of administration, as this can be done by ICU staff rather than necessitating transport to a dialysis unit. Additionally, CRRT can be run 24 hours per day [3]. While most reports describe the use of CRRT in patients with acute kidney injury (AKI), there are few reports suggesting their utility in cases of salicylate toxicity.

\section{Case Report}

In a self-harm attempt, an 18 year-old female overdosed on an unknown quantity of salicylate prior to jumping in front of traffic and being struck by a vehicle at high velocity. She was reported to have a GCS of 3 on the scene,

*The authors report no conflicts of interest. and was immediately intubated by paramedics and transferred to a level 1 trauma center.

Her trauma evaluation revealed multiple injuries including a subdural hematoma, renal laceration, multiple rib fractures, pulmonary contusions, and several long bone fractures. Initially, medication overdose was neither suspected nor screened for. She was admitted to the trauma ICU and soon after became hyperthermic (38.9 C) and hypotensive (85/41).

Three hours into her trauma ICU course, after investigation by the trauma department's social worker, screening for common xenobiotics of abuse was undertaken. A serum salicylate concentration of $24.7 \mathrm{mg} / \mathrm{dL}$ (therapeutic range $10-25 \mathrm{mg} / \mathrm{dL}$ ) was noted (the remaining toxicology screening was negative, although she may have co-ingested aripiprazole as well). An arterial blood gas at the same time noted: $\mathrm{pH} 7.08, \mathrm{pCO}_{2} 71 \mathrm{mmHg}, \mathrm{pO}_{2} 42.1$ $\mathrm{mmHg}$ and $\mathrm{HCO}_{3} 19 \mathrm{mmol} / \mathrm{L}$ while ventilated on $100 \%$ $\mathrm{FiO}_{2}$. Her serum creatinine was $1.52 \mathrm{mg} / \mathrm{dL}$. A sodium bicarbonate drip was initiated (3 amps of D50 added to 1L of D5 Normal Saline at $150 \mathrm{~mL} / \mathrm{hr}$ ) and she received 2 doses of activated charcoal ( $50 \mathrm{~g}$ ) via nasogastric tube. Despite normalization of serum bicarbonate levels and aggressive ventilator management, the patient had refractory respiratory acidosis. Over the next 8 hours, the salicylate concentrations rose to $45.2 \mathrm{mg} / \mathrm{dL}$. (Course of vitals and labs are summarized in Table 1). We felt 
Table 1. Clinical course of vitals and labs.

\begin{tabular}{lcccccccc}
\hline & $\mathrm{Tmax}$ & $\mathrm{BP}$ & $\mathrm{pH}$ & $\mathrm{pCO}_{2}$ & $\mathrm{pO}_{2}$ & $\mathrm{HCO}_{3}^{-}$ & $\mathrm{Cr}$ & Salicylate level \\
\cline { 2 - 8 } 0 hours & $38.9^{\circ} \mathrm{C}$ & $85 / 41$ & & & & & & \\
3 hours & & & 7.08 & $71 \mathrm{mmHg}$ & $42.1 \mathrm{mmHg}$ & $19 \mathrm{mmol} / \mathrm{L}$ & $1.52 \mathrm{mg} / \mathrm{dL}$ & $24.7 \mathrm{mg} / \mathrm{dL}$ \\
11 hours & & & & & & & & $45.2 \mathrm{mg} / \mathrm{dL}$ \\
\hline
\end{tabular}

Tmax = maximum temperature; $\mathrm{BP}=$ blood pressure; $\mathrm{pCO}_{2}=$ partial pressure of $\mathrm{CO}_{2} ; \mathrm{pO}_{2}=$ partial pressure of $\mathrm{O}_{2} ; \mathrm{HCO}-=$ bicarbonate; $\mathrm{Cr}=$ creatinine.

this reflected both continuous absorption (despite two dose of activated charcoal) and decreased renal elimination (likely from persistent acidemia and aciduria). While a salicylate concentration of $45.2 \mathrm{mg} / \mathrm{dL}$ would not prompt dialysis in most cases, the upward trend and inability to control serum $\mathrm{pH}$ was concerning. Nephrology was consulted with a request to initiate dialysis.

The decision to use continuous veno-venous hemodiafiltration (CVVHDF), a form of continuous renal replacement therapy (CRRT), rather than HD was considered due to the patient's head injury, hypotension and the relatively low concentrations of serum salicylate. Owing to slow clearance, CRRT in general has a limited role in most acute overdose scenarios. In this case, the multispecialty team felt the risk of worsening traumatic brain injury was greater than the risk of slower salicylate clearance. A blood flow rate of $150 \mathrm{~mL} / \mathrm{min}$ was chosen, yielding a salicylate clearance rate of $7.5 \mathrm{~mL} / \mathrm{min}$ with an extraction ratio of 5 . At this rate, it took approximately 48 hours to completely clear salicylate.

\section{Discussion}

Broadly speaking, CRRT currently has a limited role in toxicology due to the slower xenobiotic clearance. There is a paucity of data reporting clearance rates with CRRT compared to conventional HD for most toxins, including salicylates. While there is evidence that CRRT is better tolerated than HD in the hemodynamically unstable patient, whether there is an impact on patient outcome is debated [2,5]. Wrathall, et al. reports 3 cases suggesting benefits of using CRRT for Salicylate toxicity particularly in cases of severe salicylate toxicity with no available HD or in the hemodynamically unstable [6]. Our case further supports the use of CRRT, particularly in the hemodynamically unstable trauma patient with salicylate overdose of unknown quantity. We posit that in situations where HD may have an unfavorable risk profile, and time allows, CRRT may be considered as an alternative modality of enhanced elimination. We report successful clearance of salicylates with a clearance rate of $7.5 \mathrm{~mL} / \mathrm{min}$ and an extraction ratio of 5 when continuous veno-venous hemodiafiltration is utilized.

\section{REFERENCES}

[1] P. Kramer, W. Wigger and J. Rieger, “Arteriovenous Hemofiltration: A New and Simple Method for the Treatment of Overhydrated Patients Resistant to Diuretics,” Journal of Molecular Medicine, Vol. 55, No. 22, 1977, pp. 1121-1122. doi:10.1007/BF01477940

[2] M. S. Joy, G. R. Matzke, D. K. Armstrong, M. A. Marx, and B. J. Zarowitz, "A Primer on Continuous Renal Replacement Therapy for Critically Ill Patients,” Annals of Pharmacotherapy, Vol. 32, No. 3, 1998, pp. 362-75. doi:10.1345/aph.17105

[3] J. W. Goodman and D. S. Goldfarb, "The Role of Continuous Renal Replacement Therapy in the Treatment of Poisoning," Seminars in Dialysis, Vol. 19, No. 5, 2006, pp. 402-407. doi:10.1111/j.1525-139X.2006.00194.X

[4] Z. Kim and D. S. Goldfarb, “Continuous Renal Replacement Therapy Does Not Have a Clear Role in the Treatment of Poisoning," Nephron Clinical Practice, Vol. 115, No. 1, 2010, pp. c1-c6. doi:10.1159/000286343

[5] P. K. Tyagi, J. F. Winchester and D. A. Feinfeld, "Extracorporeal Removal of Toxins,” Kidney International, Vol. 74, No. 10, 2008, pp. 1231-1233. doi:10.1038/ki.2008.476

[6] G. Wrathall, R. Sinclair, A. Moore and D. Pogson, "Three Case Reports of the Use of Haemodiafiltration in the Treatment of Salicylate Overdose," Human \& Experimental Toxicology, Vol. 20, No. 9, 2001, pp. 491-495. doi:10.1191/096032701682693071 